\title{
Can lifestyle influence genetics?
}

\author{
Beth Y Karlan
}

They say "you are what you eat", but can diet and exercise alter the fate predetermined by our genetics? BRCA1 and BRCA2 mutations are the strongest risk factors for developing breast and ovarian cancers. For women who have inherited one of these mutations, the lifetime risk approaches $80 \%$ for breast cancer and $15-60 \%$ for ovarian cancer. ${ }^{1,2}$ Variations in penetrance suggest that factors other than gene mutations may influence the risk of cancer in susceptible individuals. There is mounting evidence to link body mass index (BMI) and level of physical activity with breast and ovarian cancer risk. ${ }^{1,3}$ Are there molecular mechanisms linking these lifestyle factors with $B R C A$-associated cancer penetrance?

The current mainstay of cancer prevention in $B R C A$ carriers is prophylactic surgery (i.e. bilateral mastectomy and salpingo-oophorectomy) and/or hormonal interventions such as oral contraceptives and tamoxifen. ${ }^{2}$ A preventative role for diet and exercise would provide an attractive addition to the current strategies.

Adipose tissue has a direct effect on the synthesis and bioavailability of sex steroids, and increases the production of insulin and insulin-like growth factors (IGFs). Functional interactions between these obesity-related endocrine factors and BRCA-encoded proteins have been reported. Deleterious alterations in $B R C A$ genes result in an unstable genetic environment leading to tumorigenesis. ${ }^{1}$ Why $B R C A$ mutations predispose specifically to breast and ovarian cancers remains uncertain, but the hormonal milieu of these tissues has been implicated.

Estrogen influences the normal development of breast tissue and modulates the risk of breast cancer. The relationship between obesity and breast cancer is thought to be mediated by excess estrogens produced by adipocytes. ${ }^{3}$ Even though BRCA1-associated breast cancers arise primarily from basal cells that are estrogen-receptor (ER)-negative, these cells are influenced by neighboring ER-positive epithelial cells. ${ }^{4}$ Obesity increases the expression of the endogenous estrogens that drive the growth of
"When

coupled with

dysregulated

$B R C A$ function, excess body

weight may

exacerbate the

risk of breast

or ovarian

cancer."

BY Karlan is an Advisory Board member of Nature Clinical Practice Oncology.

\section{Competing interests}

The author declared associations with the following companies:

Amgen, Bayer and

Genentech. See the article online for full details of the relationships.

www.nature.com/clinicalpractice doi:10.1038/ncponc0951 epithelial cells, which in turn induce proliferation of the basal cells. The combined effect of estrogenpromoted cell proliferation coupled with the loss of the BRCA-DNA repair system ultimately leads to carcinogenesis. Avoiding excess body weight may reduce breast cancer penetrance.

Obesity is associated with an increased risk of ovarian cancer, and is an independent predictor of disease-free survival and overall survival. ${ }^{5,6}$ High $\mathrm{BMI}$ is also associated with increased serum testosterone and insulin. Over $90 \%$ of cases of ovarian cancer express androgen receptors. Dysregulated androgen responses have been observed in ovarian epithelial cells in women with BRCA1 or $B R C A 2$ mutations. ${ }^{7}$ Androgen exposure induces high levels of gene products that have been associated with shorter time to recurrence and reduced survival in patients with ovarian cancer.

Insulin and IGFs are highly expressed in obese individuals, and estrogen stimulates the expression and activity of the IGF axis components, which correlates with an increased risk of breast cancer. ${ }^{8}$ IGF2 is overexpressed in ovarian cancers and is an independent predictor of poor survival. IGF1 enhances BRCA1 protein levels, and BRCA1 reduces expression of the IGF1 receptor protein. This loop is disrupted when mutant BRCA1 de-represses the IGF1 receptor and enhances the mitogenic effects of locally produced or circulating IGFs. ${ }^{9}$

The obesity epidemic is associated with an increase in mortality from many causes, including cardiovascular disease, diabetes and cancer. When coupled with dysregulated BRCA function, excess body weight may exacerbate the risk of breast or ovarian cancer. The International Agency for Research on Cancer has concluded that avoiding weight gain has cancer prevention effects. ${ }^{3}$ Exercise and weight control should be incorporated into cancer prevention strategies for $B R C A$ mutation carriers.

Supplementary information in the form of a reference list is available on the Nature Clinical Practice Oncology website. 